\title{
Review of: "Low incidence of daily active tobacco smoking in patients with symptomatic COVID-19"
}

Konstantinos Farsalinos ${ }^{1}$

1 University of West Attica

I would suggest the authors to upload their tables as images because they are long and cannot be read in the pdf format of the manuscript. 